\title{
Slow Progressing Localized Tumor Like Pharyngeal Mass: What Is Your Diagnosis?
}

\author{
S Touihmi*, A Sadki, A Lachkar, F Elayoubi, K Serraj and MR Ghailan \\ Department of otorhinolaryngology head and neck surgery, Department of internal medicine, University hospital Mohamed VI, Oujda Morocco
}

*Corresponding author: S Touihmi, Department of otorhinolaryngology head and neck surgery, Department of internal medicine, University hospital Mohamed VI, Oujda Morocco.

\begin{abstract}
Introduction: Amyloidosis is an idiopathic disorder characterized by the extracellular deposition of normally soluble proteins in an abnormal fibrillar form. Pharyngeal involvement is rare.

Case summary: 24 years old man consulted for chronic bilateral nasal obstruction, associated with upper dysphagia to solids. Nasal endoscopy found multiples masses involving the the pharynx. A cervical computed tomography (CT) scan of the neck had shown tissular heterogeneous process infiltrating the walls of the nasopharynx, oropharynx: soft palate, and the anterior wall of the hypopharynx. A direct laryngoscopy under general anesthesia was realized. The anatomopathological examination of the hypopharyngeal biopsy sample found amorphous acellular interstitial deposits, marked by Congo red, showing yellow-green apple birefringence under polarized light. Immunohistochemistry diagnosed type AL amyloidosis. The amyloidosis assessment to rule out systemic involvement showed no abnormalities. The patient did well over the next several months, but recurrence of the pharyngeal amyloid tumor necessitated additional conservative endoscopic excisions in the 7 months follow up.

Discussion: Amyloidosis in the head and neck is a rare and benign condition that usually takes the form of localized amyloidosis, Pharyngeal involvement is rare. Clinical symptomatology and imaging are poorly specific for pharyngeal involvement, macroscopically, the diagnosis is anatomopathological. Given the rarity of this disease, it is essential to rule out systemic involvement. Amyloid tumors in the pharynx are difficult to treat and commonly recur despite surgical excision.
\end{abstract}

\section{Case Report}

A 24 years man consulted in our medical structure for chronic bilateral nasal obstruction, associated with upper dysphagia to solids, dysphonia progressively evolving over 3 years, there was neither dyspnea nor symptoms of gastro esophageal reflux. The patient denied any constitutional symptoms, or weight loss, and he was otherwise healthy. There were no particular features in his medical history and family history was also insignificant.

On clinical examination of the mouth found poor buccodental hygiene. The oropharynx was otherwise normal, nasal endoscopy found multiples masses involving the anterior and lateral walls of the pharynx: starting just below the Eustachian tube orifice, reaching until the hypopharynx (Figure 1a). The submucosal masses were polypoidal, firm, yellowish, irregular and painless. The voluminous mass occupied the hypophayrnx, projecting over the glottic lumen, the overlying mucosa was smooth and irregular. The examination of the vocal cords was not possible; the mucosa of the pharynx was pale pinkish. Clinical examination of the rest of the organs showed no abnormal features, the rest of the otolaryngological examinations were normal. No lymph nodes founded.

A cervical computed tomography (CT) scan of the neck (Figure 1b) had shown tissular heterogeneous process infiltrating the walls of the nasopharynx, oropharynx: soft palate, and the anterior 
wall of the hypopharynx, the process contains calcifications, and it doesn't uptake contrast .The process diameter is $41 \mathrm{~mm}$ and, it's extended in a $11 \mathrm{~cm}$ of length. In the hypopharynx, the lesion is overhanging the glottis lumen. A biological assessment showed no abnormalities.

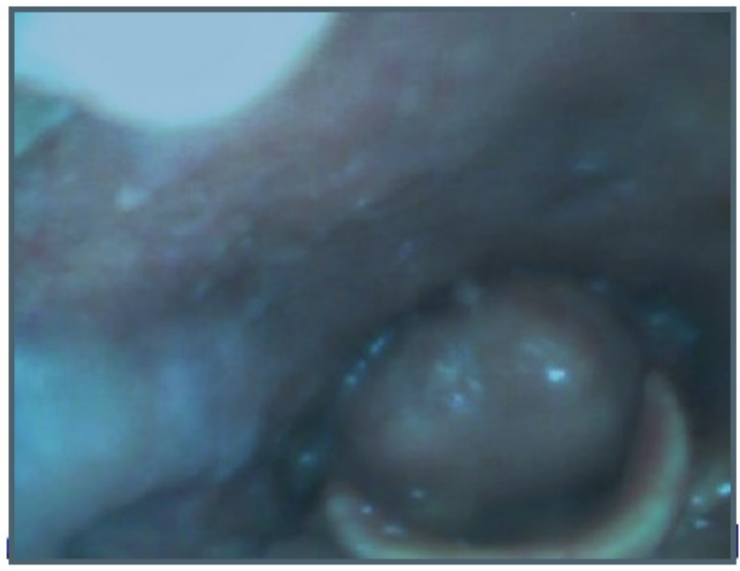

Figure 1a: Nasal endoscopy: hypopharyngeal the mass is projecting over the glottis lumen.

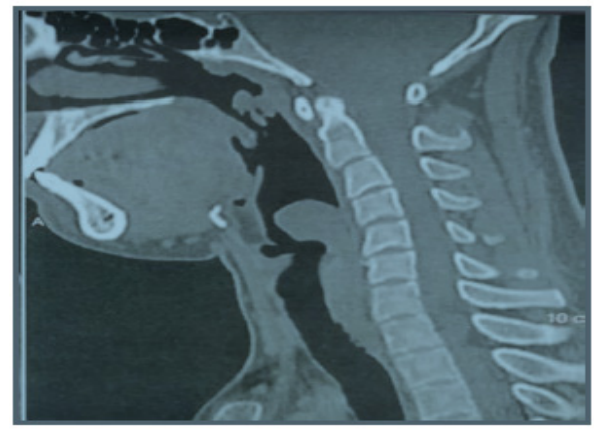

Figure 1b: Cervical CT-scan, sagittal slice(a) showing Posterior hypopharyngeal wall tissue mass extending to isodense esophagus mouth.

\section{Response}

A direct laryngoscopy under general anesthesia was realized for mainly a diagnosis but also therapeutic purpose, to free the upper airways, and so excisional biopsy of the hypopharyngeal mass and biopsy of the nasopharyngeal and oropharyngeal masses were realized. Anatomopathology of the hypopharyngeal biopsy sample found amorphous acellular interstitial deposits, marked by Congo red, showing yellow-green apple birefringence under polarized light, as is typical in amyloidosis, with no neoplastic cells or tuberculoid granuloma. Immunohistochemistry diagnosed type AL amyloidosis.

In the amyloidosis assessment to rule out systemic involvement demonstrated: The blood count found no abnormalities in the hemoglobin rate, the platelet or red or white blood-cell lines. The urine contained $0.10 \mathrm{~g} / \mathrm{l}$ of proteins; normal serum calcium and serum proteins, no peak on protein electrophoresis and an erythrocyte sedimentation rate of $4 \mathrm{~mm}$ at one hour. The result of serum immunoglobulin was normal, Urinary Ummunofixation electrophoresis was negative for Bence Jones protein with, but showed a high level of light chains kappa. Serologic tests were conducted, the results of $\mathrm{Rh}$ factor, and antinuclear antibody tests were negative. showed Bone marrow biopsy was also normal with less than $5 \%$ of plasma cell. The findings of imaging studies including echocardiogram, radiologic skeletal survey, and CT scan of thorax and abdomen were also normal. After multidisciplinary staff with the hematologist, the diagnosis was of localized ALtype amyloidosis after the patient received chemotherapy. The patient did well over the next several months, but recurrence of the pharyngeal amyloid tumor necessitated additional conservative endoscopic excisions in the 7 months follow up.

\section{Discussion}

Amyloidosis is an idiopathic disorder characterized by the extracellular deposition of normally soluble proteins in an abnormal fibrillar form. Clinically, amyloidosis can be classified as systemic and localized based on its distribution, and these two categories are further divided according to the type of the amyloid fibril protein 3 forms : AL amyloidosis, which is associated with primary systemic amyloidosis, multiple myeloma or plasma cell dyscrasias, AA or reactive (secondary) amyloidosis, which occurs in patients with chronic inflammatory conditions or neoplastic 
disease; and familial amyloidosis [1-3]. Amyloidosis in the head and neck is a rare and benign condition that usually takes the form of localized amyloidosis, Pharyngeal involvement is rare, with only 18 cases reported in the literature since 1935 mostly nasopharyngeal [3],the patients presented either with nasal obstruction as it was the case with our patient, Eustachian tube dysfunction or dysphagia. Involvement of the three section of the pharynx makes this case extremely rare: extensive search of the literature retrieved no case.

Clinical symptomatology and imaging are poorly specific for pharyngeal involvement, macroscopically, the infiltrated organs have elastic and firm consistency, but it has been described to be irregular in some few other cases as it was in our case. CT or MRI are generally useful determine the form of amyloidosis by identifying the infiltration of other organs, and also to guide treatment by defining the extension of the lesions. A homogeneous submucosal mass with only slight if any contrast uptake, is the most usual radiologic aspect of the amyloidosis lesion. There is sometimes perilesional calcification. No bone erosion neither intracranial extension nor cervical adenopathy [4] are generally present [5]. The diagnosis is anatomopathological: the deposits are extracellular amorphous eosinophilic matter, with a marked chronic inflammatory cell infiltrate. Apple green birefringence was seen when histologic sections stained with Congo red were viewed with polarized light, and Immunohistochemistry revealed AL type amyloidosis.

Given the rarity of this disease, it is essential to rule out systemic involvement. In our case the patient presented a localized form of amyloidosis, urine and serologic evaluations, as well as chest x-ray, revealed no evidence of systemic disease. No documentation exists to suggest that localized amyloidosis can progress to systemic amyloidosis [6]. Nevertheless, all patients with localized disease should be followed up for any evidence of systemic amyloidosis or recurrence. Amyloid tumors in the pharynx are difficult to treat and commonly recur despite surgical excision, Simpson et al have suggested less recurrence following laser excision of localized amyloidosis [6]. Because localized amyloidosis is a disease of great rarity and slow progression for which spontaneous regression is known to occur, it is difficult to predict the outcome [6-14] routine surveillance and follow up of the patient is necessary for recurrence.

\section{Acknowledgement}

None.

\section{Conflict of interest}

The authors declare that they have no competing interest.

\section{References}

1. M Ulmansky (1962) Primary Amyloidosis of Oral Structures and The Pharynx. Oral Surgery, Oral Medicine, Oral Pathology 15 (7): 800-802.

2. Essakali, I Rkain I Elamouri L (2016) Tongue Nodule: What Is Your Diagnosis? Rabat.

3. Edmund Pribitkin, Oren Friedman, Brian O’Hara, Mary F Cunnane, David Levi, et al. (2003) Amyloidosis of the upper aerodigestive tract. Laryngoscope 113(12): 2095-2101.

4. Carla R Penner, Susan Muller (2006) Head and neck amyloidosis: A clinicopathologic. Manitoba, Canada.

5. Naresh K Panda, Karuppiah Saravanan (2007) Localized amyloidosis masquerading as nasopharyngeal tumor: a review.

Am J Otolaryngol 28(3): 208-211.

6. Alpen Patel, Stefan Pambuccian, Robert Maisel (2002) Nasopharyngeal Amyloidosis.

7. Kian Karimi, Neil N Chheda (2010) Nasopharyngeal Amyloidosis: A Case Report Laryngoscope 4: S197.

8. Aaron D Friedman, Rajendra Bhayani, Lorenzo Memeo, Daniel B Kuriloff (2002) Localized laryngeal amyloidosis. Otolaryngol Head Neck Surg 127(5): 487-489.

9. Kristen S. Fahrner, Candice C Black (2004) Localized Amyloidosis of the Tongue: A Review. Am J Otolaryngol 25(3): 186-189.

10. Wioletta Pietruszewska1, Małgorzata Wągrowska Danilewicz, Janusz Klatka (2014) Amyloidosis of the head and neck: a clinicopathological study of cases with long-term follow-up. Arch Med Sci 10(4): 846-852.

11. Christopher R Grindle, Joseph M Curry, Joshua P Cantor (2011) Localized oropharyngeal amyloidosis. Ear Nose Throat J 90(5): 220-222.

12. B Hammami, M Mnejja, S Kallel, L Bouguecha, A Chakroun (2010) Hypopharyngeal amyloidosis: A case report. Eur Ann Otorhinolaryngol Head Neck Dis 127(2): 83-85.

13. Ashley O’Reilly, Anita D’Souza, John Lust (2013) Localized Tongue Amyloidosis: A Single institutional case series Otolaryngol Head Neck Surg 149(2): 240-244.

14. Deveze A, Yakoot S, Giusiano M, Sanjuan A, Mardassi J, Magnan A (2010) Une Cause Rare D Otite Seromuqueuse. 